\title{
ON THE DEFICIENCIES OF COMPOSITE ENTIRE FUNCTIONS
}

\author{
by J. K. LANGLEY*
}

(Received 17th June 1991)

\begin{abstract}
For any sequence $\left(a_{j}\right)$ of complex numbers and for any $\rho>1 / 2$, we construct an entire function $F$ with the following properties. $F$ has order $\rho$, mean type, each $a_{j}$ is a deficient value of $F$, and $F$ is given by $F(z)=f(g(z))$, where $f$ and $g$ are transcendental entire functions. This complements a result of Goldstein. We also construct, for any $\rho>1 / 2$, an entire function $G$ of order $\rho$, mean type, such that $\liminf _{r \rightarrow \infty} T(r, G) / T\left(r, G^{\prime}\right)>1$.
\end{abstract}

1991 Mathematics subject classification: 30D35, Secondary 30D05, 30D20.

\section{Introduction}

We are concerned with the deficiencies of an entire function $F$ given by $F(z)=f(g(z))$, where $f$ and $g$ are transcendental entire functions. It is well-known [9, p. 53] that if $F$ has finite order, then $f$ must have order zero, which implies that $F$ has no finite Picard value. The following was proved by Goldstein [5] (see also [6]). Here the notation is that of [9], which we shall use throughout.

Theorem A. Suppose that $f$ and $g$ are transcendental entire functions such that $F(z)=f(g(z))$ has finite order. Then

$$
\sum_{a} \delta(a, F)<1
$$

where the sum is taken over all finite values a.

We remark that the hypothesis that $F$ has finite order is necessary in Theorem $A$ because of the obvious example $F=e^{g}$, which has infinite order if $g$ is transcendental entire. The proof of Theorem A depends heavily on results of Edrei and Fuchs [3], and in particular on the fact that if $F$ is entire of finite order with maximal deficiency sum, then each deficient value of $F$ is an asymptotic value of $F$. As remarked in [5], it is possible to replace 1 on the right-hand-side of (1.1) by a constant slightly smaller than 1 , but depending on the lower order of $F$ (see [3]).

In the terminology of factorization theory (see $[7,17]$ ), Theorem $A$ states that an entire function $F$ of finite order with maximal deficiency sum is pseudoprime, that is, it

* Research supported in part by a grant from the Alexander von Humboldt Stiftung. 
has no factorization $F(z)=f(g(z))$, where $f$ and $g$ are transcendental entire. The following question appears in [17], and is attributed to Fuchs and Song. If $F$ is entire of finite order with $\delta(a, F)>0$ for some finite $a$, must $F$ be pseudoprime? We answer this question in the negative, by constructing a non-pseudoprime entire function of finite order with infinitely many deficient values.

Now the following was proved by Arakelyan [1].

Theorem B. For any sequence $\left(a_{j}\right)$ of finite complex numbers, and for any $\rho>1 / 2$, there exists an entire function $G$ of order $\rho$, mean type, such that for each $j, \delta\left(a_{j}, G\right)>0$.

No such result is possible for $\rho \leqq 1 / 2$ (see [9, Ch. 4]). We remark that Eremenko [4] showed further that the function $G$ may be constructed so as to have the finite deficient values $a_{j}$, but no other finite deficient values. Using in part methods similar to those of Arakelyan, we shall prove the following.

Theorem 1. For any sequence $\left(a_{k}\right)(k=0,1,2, \ldots)$ of complex numbers, and for any $\sigma$ with $1 / 2<\sigma<+\infty$, there exist transcendental entire functions $f$ and $g$ such that $F(z)=f(g(z))$ has order $\sigma$, mean type, and such that for each $k, \delta\left(a_{k}, F\right)>0$.

As remarked by Arakelyan in [1], it is only necessary to prove Theorem 1 for $1 / 2<\sigma \leqq 1$, for otherwise we need only consider, for a suitable value of $N$, the function $G(z)=F\left(z^{N}\right)=f\left(g\left(z^{N}\right)\right)$, which has the same deficiencies as $F$. To construct a composite entire function $F$ of order $\sigma$ having $\delta(0, F)>0$, we can take $\delta, \rho$ such that $(1+\delta) \rho=\sigma$, and form $f$ as a Weierstrass product satisfying $T(r, f)=0(\log r)^{1+\delta}$. The function $f$ has zeros of large multiplicity at the points $\exp \left(\lambda^{n}\right)$, where $\lambda$ is large and positive. We then construct $g$, using a simplified version of Arakelyan's method, so that $T(r, g)=0\left(r^{\rho}\right)$ and so that $\left|g(z)-\exp \left(\lambda^{n}\right)\right|<1$ in a subset of an annulus $\lambda^{m} \leqq|z| \leqq \lambda^{m+1}$. To construct a function $F$ having infinitely many deficient values, we apply a transformation used in [2] to quasiconformally modify $f$ in successive annuli so that for each $k$ and for infinitely many $n, f(z)-a_{k}$ has a zero of large multiplicity close to $\exp \left(\lambda^{n}\right)$. It does not seem possible, however, to prove by our method that the $a_{k}$ are the only finite deficient values of $F$.

Our construction of $g$ also has a bearing on the following problem. If $f$ is a function meromorphic and transcendental in the plane, it is well-known [9] that the derivative $f^{\prime}$ satisfies

$$
T\left(r, f^{\prime}\right) \leqq(2+o(1)) T(r, f)
$$

at least outside a set of $r$ of finite measure, and that $2+o(1)$ may be replaced by $1+o(1)$ if $f$ is entire. In the other direction it was recently proved by Hayman and Miles [10] that for any transcendental meromorphic function $f$ and for any $K>1$, $T(r, f) \leqq 3 e K T\left(r, f^{\prime}\right)$ for $r$ lying in a set of positive lower logarithmic density. In particular

$$
L(f)=\liminf _{r \rightarrow \infty} T(r, f) / T\left(r, f^{\prime}\right) \leqq 3 e
$$


Now it was proved by Toppila in [16] that if $f$ is transcendental and meromorphic of order zero, then $L(f)$ as defined by (1.3) is at most 1. On the other hand Toppila constructed in [16] and [15] meromorphic functions of arbitrary finite positive order and an entire function of order 1 such that $L(f)>1$. Our construction of $g$ leads to the following.

Theorem 2. For any $\rho$ with $1 / 2<\rho<+\infty$, there exists an entire function $g$ of order $\rho$, mean type, such that

$$
\liminf _{r \rightarrow \infty} T(r, g) / T\left(r, g^{\prime}\right) \geqq K(\rho)>1
$$

Again it is only necessary to prove Theorem 2 for $1 / 2<\rho \leqq 1$. In our construction, $K(\rho) \rightarrow 1$ as $\rho \rightarrow 1 / 2$, which suggests the possibility that if $g$ is transcendental entire of order $\rho \leqq 1 / 2$, then $L(g)=1$.

\section{Lemmas needed for the proofs of Theorems 1 and 2}

The following Lemmas A and B are due to Keldysh and play the same role as in Arakelyan's construction [1].

Lemma A [14]. There exist positive constants $c_{1}, c_{2}$ with the following property. Suppose that $L$ is a rectifiable path of length $S$ joining the points $a$ and $b$, and that $\varepsilon$ and $d$ are positive. Then there is a polynomial $P$ such that

$$
|P(1 /(z-b))-1 /(a-z)|<\varepsilon
$$

for all $z$ such that dist $\{z, L\} \geqq d$, and

$$
|P(1 /(z-b))|<\exp \left(c_{1}(1+|\log \varepsilon d|) \exp \left(c_{2} S / d\right)\right)
$$

for all $z$ with $|z-b| \geqq d$.

Here dist $\{z, L\}=\inf \{|z-w|: w$ on $L\}$.

Lemma B [13]. Suppose that $1 / 2<\rho \leqq 1$ and $0<\alpha<\pi-\pi / 2 \rho$. Suppose further that $H$ is analytic in $\operatorname{Re}(z) \geqq 0$, and satisfies $\log ^{+}|H(z)|=0\left(1+|z|^{\rho}\right)$ there. Then there exists an entire function $g$ of at most order $\rho$, mean type, such that $|g(z)-H(z)|<1 / 2$ for all large $z$ satisfying $|\arg z| \leqq \alpha$.

The following lemma gives us some control over the rate at which the deficiencies $\delta\left(a_{k}, F\right)$ tend to zero as $k \rightarrow \infty$ in Theorem 1 . The idea for the proof was suggested by the author's colleague, D. A. Burgess.

Lemma 1. Let $\alpha_{0}, \alpha_{1}, \ldots$, be positive real numbers such that $\sum_{k=0}^{\infty} \alpha_{k}<1$. Then there 
are pairwise disjoint sets $T_{k}$ of non-negative integers such that each $T_{k}$ is an arithmetic progression modulo $p_{k}$, where $p_{k}$ satisfies $1 \leqq p_{k} \leqq 2 / \alpha_{k}$.

Proof. For each $k$ we choose a positive integer $i_{k}$ such that $1 / \alpha_{k} \leqq 2^{i_{k}} \leqq 2 / \alpha_{k}$, and we set $p_{k}=2^{i_{k}}$. By a rearrangement if necessary, we may assume that the sequence $\left(i_{k}\right)$ is non-decreasing. We set $y_{0}=0$, and $T_{0}=\left\{n p_{0}: n=0,1,2, \ldots\right\}$.

Suppose now that disjoint sets $T_{0}, \ldots, T_{m}$ have been chosen, such that each $T_{k}$ is an arithmetic progression modulo $p_{k}$, with first term $y_{k} \leqq p_{k}$. Now if $k \leqq m$, the number of elements of the set $\left\{1,2, \ldots, p_{m+1}\right\}$ which are congruent to $y_{k}$ modulo $p_{k}$ is $p_{m+1} / p_{k}$. So the number of elements of the set $\left\{1, \ldots, p_{m+1}\right\}$ which are congruent to $y_{k}$ modulo $p_{k}$ for some $k \leqq m$ is at most

$$
p_{m+1}\left(\sum_{k=0}^{m} 1 /\left(p_{k}\right)\right) \leqq p_{m+1}\left(\sum_{k=0}^{m} \alpha_{k}\right)<p_{m+1}
$$

So there is some $y_{m+1}$ in the set $\left\{1, \ldots, p_{m+1}\right\}$ which is not congruent to $y_{k}$ modulo $p_{k}$ for any $k \leqq m$, and we set $T_{m+1}=\left\{y_{m+1}+n p_{m+1}: n=0,1,2, \ldots\right\}$. In this way the sets $T_{k}$ are defined inductively. Now if $k<m$ and the sets $T_{k}$ and $T_{m}$ are not disjoint, then $y_{m}$ is congruent to $y_{k}$ modulo $p_{k}$, which is impossible.

\section{Construction of the function $g$}

Our construction of the function $g$ in the composition $F(z)=f(g(z))$ is based on the following lemma, which in turn is based on Arakelyan's method in [1].

Lemma 2. Let $1 / 2<\rho \leqq 1$ and $1<\lambda<+\infty$. Then there exist positive sequences $\left(\theta_{k}\right)$, $\left(\alpha_{k}\right),\left(n_{k}\right)$ and $\left(\varepsilon_{k}\right)$ with the following properties.

$$
\begin{gathered}
\sum_{k=0}^{\infty} \alpha_{k}<1 \\
\varepsilon_{k}=\exp \left(-1 /\left(\delta_{2} \alpha_{k}\right)\right) \text { for each } k
\end{gathered}
$$

where $\delta_{2}$ is a positive constant.

(iii) If $\beta_{k, n}$ are any constants satisfying

$$
\log ^{+}\left|\beta_{k, n}\right| \leqq \varepsilon_{k} \lambda^{n \rho}
$$

then there exist a positive $N_{0}$ and an entire function $g$ of at most order $\rho$, mean type, such that if $n \geqq \max \left\{n_{k}, N_{0}\right\}$, we have

$$
\left|g(z)-\beta_{k, n}\right|<1
$$


for $z$ satisfying

$$
\frac{7}{8} \lambda^{n} \leqq|z| \leqq \frac{9}{8} \lambda^{n+1}, \quad\left|\arg z-(-1)^{n} \theta_{k}\right| \leqq 2 \alpha_{k} .
$$

We remark that in Arakelyan's construction, $\beta_{k, n}=a_{k}$ for all $k$ and $n$, and that since the conclusion of (3.4) is weaker than that required in Arakelyan's construction, we are able to dispense with the infinite product $\omega$ of [1, Lemma 3].

To prove Lemma 2, we take a positive $\alpha^{*}$ such that

$$
\alpha^{*}<\pi-\pi / 2 \rho
$$

and we choose an infinite sequence $\left(\theta_{k}\right)$ satisfying

$$
0<\theta_{0} \leqq \theta_{k}<\theta_{k+1}<\alpha^{*}
$$

for $k \geqq 0$. We choose positive constants $\alpha_{k}$ such that $\theta_{0}-8 \alpha_{0}>0$ and such that the intervals $\left(\theta_{k}-8 \alpha_{k}, \theta_{k}+8 \alpha_{k}\right)$ are disjoint, and note that (3.1) is satisfied. We form, for $k \geqq 0$ and $n \geqq 1$, regions

$$
E_{k, n}=\left\{z: \frac{3}{4} \lambda^{n} \leqq|z| \leqq \frac{5}{4} \lambda^{n+1},\left|\arg z-(-1)^{n} \theta_{k}\right| \leqq 4 \alpha_{k}\right\}
$$

and

$$
F_{k, n}=\left\{z: \frac{7}{8} \lambda^{n} \leqq|z| \leqq \frac{9}{8} \lambda^{n+1},\left|\arg z-(-1)^{n} \theta_{k}\right| \leqq 2 \alpha_{k}\right\}
$$

We note that $F_{k, n}$ is contained in $E_{k, n}$, and that the $E_{k, n}$ are disjoint and for fixed $k$ lie alternately above and below the real axis.

Now let $Y_{k, n}$ be the boundary of $E_{k, n}$. We join $Y_{k, n}$ to the point $-\lambda^{n+1 / 2}$ by an arc $L_{k, n}$ of the circle $|z|=\lambda^{n+1 / 2}$, taking always the shortest possible such arc. Now

$$
s_{k, n}=\text { length }\left(Y_{k, n} \cup L_{k, n}\right)<c_{1} \lambda^{n}
$$

where $c_{1}, c_{2}, \ldots$, henceforth denote positive constants which are independent of $k$ and $n$. Also

$$
\operatorname{dist}\left(F_{k, n}, Y_{k, n} \cup L_{k, n}\right)>c_{2} \alpha_{k} \lambda^{n}
$$

where $\operatorname{dist}(A, B)=\inf \{|z-w|: z$ in $A, w$ in $B\}$. We set

$$
d_{k, n}=\delta_{1} \alpha_{k} \lambda^{n}
$$

where $\delta_{1}$ is positive, but small compared to $c_{2}$, and set

$$
D_{k, n}=\left\{z: \operatorname{dist}\left\{z, Y_{k, n} \cup L_{k, n}\right\}<d_{k, n}\right\}
$$


Provided $\delta_{1}$ is small enough, the $D_{k, n}$ are disjoint for fixed $k$, and

$$
F_{k, n} \cap D_{k, n}=\phi
$$

We set

$$
\varepsilon_{k}=\exp \left(-1 /\left(\delta_{2} \alpha_{k}\right)\right)
$$

where $\delta_{2}$ is small and positive. We apply Lemma A with $d=d_{k, n}, a=u$, where $u$ lies on $Y_{k, n}$, and $b=-\lambda^{n+\frac{1}{2}}$. We obtain functions $Q_{k, n}(u, z)$, each of which is for fixed $u$ a polynomial in $1 /\left(z+\lambda^{n+\frac{1}{2}}\right)$, such that for $u$ on $Y_{k, n}$ and $z$ not in $D_{k, n}$,

$$
\left|Q_{k, n}(u, z)-1 /(u-z)\right|<\frac{16}{25} \lambda^{-2 n-2} \exp \left(-\varepsilon_{k} \lambda^{n \rho}\right)
$$

Here we have chosen $\varepsilon$ in Lemma $A$ to equal the right-hand-side of (3.16). Provided $n \geqq n^{*}(k)$, say, and $u$ lies on $Y_{k, n}, Q_{k, n}(u, z)$ is analytic in $\operatorname{Re}(z) \geqq-1$ and satisfies, for such $z$,

$$
\left|Q_{k, n}(u, z)\right|<\exp \left(c_{3} \varepsilon_{k} \lambda^{n \rho} \exp \left(c_{4} / \alpha_{k}\right)\right)<\exp \left(\lambda^{n \rho}\right)
$$

provided $\delta_{2}$ is small enough in (3.15). By an obvious compactness argument, we can assume that $Q_{k, n}(u, z)$ is piecewise constant in $u$.

Now suppose that the constants $\beta_{k, n}$ satisfy

$$
\log ^{+}\left|\beta_{k, n}\right| \leqq \varepsilon_{k} \lambda^{n \rho}
$$

and set

$$
h(z)=\beta_{k, n} \text { for } z \text { in } E_{k, n} \text {. }
$$

We choose integers $n_{k} \geqq n^{*}(k)$ so large that

$$
\sum_{k=0}^{\infty} \sum_{n=n_{k}}^{\infty} \int_{Y_{k, n}} 1 /|u|^{2}|d u|<1 / 2
$$

and we set

$$
V_{n}=\bigcup_{\substack{k, m \\ n_{k} \leqq m \leqq n}} Y_{k, m}
$$

In addition we set 


$$
H_{n}(z)=(1 / 2 \pi i) \int_{V_{n}} h(u) Q(u, z) d u,
$$

where $Q(u, z)=Q_{k, m}(u, z)$ for $\operatorname{Re}(z) \geqq-1, u$ on $Y_{k, m}$. Here all integrals are taken in the positive sense, and $H_{n}$ is analytic in $\operatorname{Re}(z) \geqq-1$.

Suppose now that $|z| \leqq \lambda^{N}$, and that $m>n>N+c_{5}$. Then

$$
\int_{V_{m} \backslash V_{n}} 1 /(u-z) d u=0
$$

and so

$$
\begin{aligned}
\left|H_{m}(z)-H_{n}(z)\right| & \leqq(1 / 2 \pi)\left|\int_{V_{m} \backslash V_{n}} h(u)\left(Q(u, z)-\frac{1}{u-z}\right) d u\right| \\
& \leqq(1 / 2 \pi) \int_{V_{m} \backslash V_{n}} 1 /|u|^{2}|d u| \rightarrow 0 \text { as } n \rightarrow \infty
\end{aligned}
$$

using (3.16), (3.18), (3.19) and (3.20). Thus

$$
H(z)=\lim _{n \rightarrow \infty} H_{n}(z)
$$

is analytic in $\operatorname{Re}(z) \geqq-1$.

Now denote by $\bar{F}$ the union of the sets $F_{k, n}$, for $k \geqq 0$ and $n \geqq n_{k}$. Then if $z$ is in $F$, and $n$ is large,

$$
(1 / 2 \pi i) \int_{V_{n}} h(u) 1 /(u-z) d u=h(z)
$$

because $z$ lies inside precisely one $Y_{k, m}$, and further, using (3.16),

$$
\begin{aligned}
\left|H_{n}(z)-h(z)\right| & =(1 / 2 \pi)\left|\int_{V_{n}} h(u)\left(Q(u, z)-\frac{1}{u-z}\right) d u\right| \\
& \leqq(1 / 2 \pi) \int_{V_{n}} 1 /|u|^{2}|d u|<1 / 4 \pi
\end{aligned}
$$

Here we have used the fact that by (3.14) $F$ does not meet any $D_{k, n}$. Thus for all $z$ in $F$,

$$
|H(z)-h(z)|<1 / 10 \text {. }
$$

Now we estimate the growth of $H$. Assume that $\lambda^{N-1} \leqq|z| \leqq \lambda^{N}$, and set $M=N+c_{6}$. If $c_{6}$ is chosen large enough then as in the proof of (3.23), $m>M$ gives $\left|H_{m}(z)-H_{M}(z)\right|<$ $1 / 4 \pi$, using (3.20). Also 


$$
\begin{aligned}
\left|H_{M}(z)\right| & \leqq(1 / 2 \pi) \int_{V_{M}} 1 /|u|^{2}|d u| \max _{u \text { in } V_{M}}|u|^{2}|h(u) Q(u, z)| \\
& =0\left(|z|^{2} \exp \left(c_{7} \lambda^{N \rho}\right)\right)
\end{aligned}
$$

using (3.17) and (3.18). This gives

$$
\log ^{+}|H(z)|=0\left(1+|z|^{p}\right) .
$$

Now using Lemma B and (3.6) and (3.7) we can choose an entire function $g$ of at most order $\rho$, mean type, such that $|g(z)-H(z)|<1 / 2$ for large $z$ with $|\arg z| \leqq \alpha^{*}$. Now using (3.19) and (3.24) we obtain (3.4). This completes the proof of Lemma 2.

\section{Proof of Theorem 2}

We choose

$$
\beta_{0, n}=\exp \left(\varepsilon_{0} \lambda^{n \rho}\right)
$$

in (3.18). Constructing $g$ as in Section 3, Cauchy's integral formula gives $\left|g^{\prime}(z)\right|<1$ for large $z$ satisfying

$$
\lambda^{n} \leqq|z| \leqq \lambda^{n+1},\left|\arg z-(-1)^{n} \theta_{0}\right| \leqq \alpha_{0}
$$

On the other hand, if $n$ is large and $z$ satisfies (4.2),

$$
\left|g(z)-\beta_{0, n}\right|<1
$$

For large $n$, and $r$ in $\left[\lambda^{n}, \lambda^{n+1}\right]$, we denote by $L_{r}$ the set of $\theta$ in $[0,2 \pi]$ such that $r e^{i \theta}$ satisfies (4.2). Then clearly

$$
(1 / 2 \pi) \int_{L_{r}} \log { }^{+}\left|g\left(r e^{i \theta}\right)\right| d \theta>c_{1} r^{\rho}
$$

for some positive constant $c_{1}$, while $L_{r}$ makes no contribution to $m\left(r, g^{\prime}\right)$. On the other hand, if $M_{r}=[0,2 \pi] \backslash L_{r}$, then

$$
(1 / 2 \pi) \int_{M_{r}} \log ^{+}\left|g^{\prime}\left(r e^{i \theta}\right)\right| d \theta \leqq(1 / 2 \pi) \int_{M_{r}} \log ^{+}\left|g\left(r e^{i \theta}\right)\right| d \theta+m\left(r, g^{\prime} / g\right)=0\left(r^{\rho}\right) .
$$

It follows at once that $g$ has order $\rho$, mean type and that

$$
L(g)=\liminf _{r \rightarrow \infty} T(r, g) / T\left(r, g^{\prime}\right)>1 .
$$


We remark that for the proof of Theorem 2 we do not in fact need the sets $F_{k, n}$ for $k \geqq 1$. However we still need to apply Lemma $\mathrm{B}$ in order to obtain the entire function $g$ from the analytic function $H$. Since Lemma B restricts the angle in which we may approximate $H$ by $g$, we see that for our examples $L(g) \rightarrow 1$ as $\rho \rightarrow 1 / 2$.

\section{The construction of $f$}

The entire function $f$ required for the proof of Theorem 2 will be constructed using the following lemma.

Lemma 3. Let $a_{0}, a_{1}, \ldots$ be any complex numbers. Let $0<\delta \leqq 1$, and let $\lambda>1$ be such that $\lambda^{\delta}$ is an integer and

$$
\lambda^{\delta}<\left(\lambda^{\delta}-1\right)(1+\delta)
$$

In addition, let $T_{0}, T_{1}, \ldots$ be pairwise disjoint infinite sets of non-negative integers. Then there exists an entire function $f$ with the following properties.

$$
\log M(r, f)=0(\log r)^{1+\delta}
$$

(ii) There exist positive constants $c$ and $n^{*}$ and a sequence $\left(B_{n}\right)$ satisfying

$$
|\log | B_{n}\left|-\lambda^{n}\right|<1
$$

for $n \geqq n^{*}$, and such that if $n \geqq n^{*}$ and $n$ is in $T_{k}$ and $\lambda^{n} \geqq \log ^{+}\left|a_{k}\right|$, we have

$$
\log \left|f(z)-a_{k}\right|<-c \lambda^{n(1+\delta)}
$$

for all $z$ with $\left|z-B_{n}\right|<1$.

Proof. We set

$$
h(z)=\prod_{n=0}^{\infty}\left(1-z / e^{\lambda^{n}}\right)^{\lambda^{n \delta}}
$$

Now if $\exp \left(\lambda^{n}\right) \leqq r<\exp \left(\lambda^{n+1}\right)$, the number of zeros of $h$ in $|z| \leqq r$ is $1+\lambda^{\delta}+\cdots+\lambda^{n \delta}$ which is less than $\lambda^{\delta}\left(\lambda^{\delta}-1\right)^{-1}(\log r)^{\delta}$. Hence the counting function $n(r, 1 / h)$ of the zeros of $h$ satisfies

$$
n(r, 1 / h)<\lambda^{\delta}\left(\lambda^{\delta}-1\right)^{-1}(\log r)^{\delta}
$$

for all positive $r$. Thus by $\left[9\right.$, p. 28] we see that $\log M(r, h)=0(\log r)^{2}$. For a more precise estimate, it follows from a result of Hayman [8] that for $r$ outside a set of finite 
logarithmic measure, $\log M(r, h) \sim T(r, h)=N(r, 1 / h)+0(1)$ so that for some $d$ with $0<d<1$ and for all large $r$ we have, using (5.1) and (5.6),

$$
\log M(r, h)<d(\log r)^{1+\delta} .
$$

Now suppose that $\left|z-\exp \left(\lambda^{n}\right)\right|=(1 / 4) \exp \left(\lambda^{n}\right)$, with $n$ large. Then (5.7) gives

$$
\begin{aligned}
\log \left|h(z)\left(z-\exp \left(\lambda^{n}\right)\right)^{-i^{n \delta}}\right| & <d\left(\log \left(\frac{5}{4} \exp \left(\lambda^{n}\right)\right)\right)^{1+\delta} \\
& -\lambda^{n \delta} \log \left(\frac{1}{4} \exp \left(\lambda^{n}\right)\right)<-8 c \lambda^{n(1+\delta)}
\end{aligned}
$$

for some positive $c$. Consequently if

$$
\log \left|z-\exp \left(\lambda^{n}\right)\right|<c \lambda^{n}
$$

we have

$$
\log |h(z)|<-7 c \lambda^{n(1+\delta)} .
$$

Now consider, for $R>0$ and $|a| \leqq R / 2$, the transformation

$$
w=w_{R}(z)=\frac{R^{2}(z+a)}{\left(R^{2}+\bar{z} a\right)}
$$

which maps $|z| \leqq R$ one-to-one onto $|w| \leqq R$, such that $w(0)=a$ and $w_{R}(z)=z$ when $|z|=R$. (This transformation is used, for example, in [2].) Also

$$
\left|w_{\bar{z}} / w_{z}\right|=\left|a w / R^{2}\right| \leqq|a| / R
$$

and

$$
|w(z)-a| \leqq 4|z|
$$

for $|z|<R$. We modify $f$ using the transformations (5.10). If $n \geqq n_{0}$ for some large $n_{0}$, we certainly have, by $[8]$,

$$
\frac{1}{2}|h(z)|>\exp \left(2 \lambda^{n}\right)=R_{n}
$$

on

$$
\left|z-\exp \left(\lambda^{n}\right)\right|=\frac{1}{4} \exp \left(\lambda^{n}\right)
$$

Suppose that $n \geqq n_{0}$, that $n$ is in the set $T_{k}$, and that $R_{n} \geqq\left|a_{k}\right|^{2}$. Then in the open disc 


$$
D_{n}=\left\{z:\left|z-\exp \left(\lambda^{n}\right)\right|<\frac{1}{4} \exp \left(\lambda^{n}\right)\right\}
$$

we set

$$
H(z)=\frac{R_{n}^{2}\left(h(z)+a_{k}\right)}{R_{n}^{2}+\overline{h(z)} a_{k}}
$$

if $|h(z)| \leqq R_{n}$, and $H(z)=h(z)$ otherwise. If $n<n_{0}$ or if $R_{n}<\left|a_{k}\right|^{2}$ or if $n$ lies in none of the sets $T_{k}$, or if $z$ lies outside the union of the discs $D_{n}$, we just set $H(z)=h(z)$.

It follows that $H$ is continuous in the plane and is analytic outside the union of the discs $D_{n}$, while in $D_{n} H$ is quasiregular with complex dilatation bounded by $R_{n}^{-1 / 2}=$ $\exp \left(-\lambda^{n}\right)$. Also for $n$ large with $n$ in $T_{k}$ and $R_{n} \geqq\left|a_{k}\right|^{2}$,

$$
\log \left|z-\exp \left(\lambda^{n}\right)\right|<c \lambda^{n}
$$

implies that

$$
\log \left|H(z)-a_{k}\right|<-6 c \lambda^{n(1+\delta)}
$$

using (5.9) and (5.12).

Let $\sigma(z)=H_{\bar{z}} / H_{z}$ be the complex dilatation of $H$, which exists almost everywhere (see [2] or [11]), and is zero outside the union of the discs $D_{n}$. Now $\sigma(z)=0(1 /|z|)$ as $z \rightarrow \infty$, by the construction of $H$, so that $\int_{|z|>1} \sigma(z) /|z|^{2} d x d y<\infty$. Proceeding as in [2], the Teichmüller-Belinskii theorem [11, p. 227] implies that there is a solution $\Lambda$ of the Beltrami equation

$$
\Lambda_{\bar{z}}=\sigma(z) \Lambda_{z}
$$

which is a quasiconformal homeomorphism of the extended plane onto itself such that $\Lambda(0)=0, \Lambda(\infty)=\infty$, and

$$
\Lambda(z)=z(1+o(1)) \quad \text { as } z \rightarrow \infty
$$

Denoting by $J=\Lambda^{-1}$ the inverse function of $\Lambda$, we note that $f(z)=H(J(z))$ is almost everywhere conformal and so entire.

Now define $B_{n}$ by

$$
B_{n}=\Lambda\left(\exp \left(\lambda^{n}\right)\right)
$$

so that (5.3) follows at once for $n$ sufficiently large, using (5.19). We consider now the distortion properties of $J=\Lambda^{-1}$. Now $J\left(B_{n}\right)=\exp \left(\lambda^{n}\right)$, and $J(z)=z(1+o(1))$, so for $n$ large set

$$
J_{n}(z)=\left(J\left(B_{n}+\frac{1}{4}\left|B_{n}\right| z\right)-e^{\lambda_{n}}\right) \exp \left(-\lambda^{n}\right)
$$


Now each $J_{n}$ maps $|z|<1$ into itself, with $J_{n}(0)=0$. Further, in terms of the real dilatation, $J_{n}$ is $K_{n}$ quasiconformal, where $K_{n}=\left(1+\kappa_{n}\right) /\left(1-\kappa_{n}\right)$, and $\kappa_{n}=0\left(1 /\left|B_{n}\right|\right)$. Distortion theorems (see, for example, [12, p. 6]) give the following. If $|z|<4 /\left|B_{n}\right|$ then we have $\left|J_{n}(z)\right| \leqq c_{1}\left(1 /\left|B_{n}\right|\right)^{1 / K_{n}}$ where $c_{1}, c_{2}$ henceforth denote positive absolute constants, so that

$$
\left|e^{\lambda n} J_{n}(z)\right| \leqq c_{2}\left|B_{n}\right|^{1-1 / K_{n}} \leqq c_{3}\left|B_{n}\right|^{c^{4}\left|B_{n}\right|-1},
$$

for such $z$. Thus $\left|z-B_{n}\right|<1$ gives

$$
\left|J(z)-e^{\lambda^{n}}\right|<\exp \left(c \lambda^{n}\right)
$$

if $n$ is large. Now (5.17), (5.18) and (5.23) imply that if $n \geqq n^{*}$, say, if $n$ is in the set $T_{k}$ and if $\lambda^{n} \geqq \log ^{+}\left|a_{k}\right|$, then $\left|z-B_{n}\right|<1$ gives

$$
\log \left|f(z)-a_{k}\right|=\log \left|H(J(z))-a_{k}\right|<-6 c \lambda^{n(1+\delta)}
$$

which proves (5.4).

To estimate the growth of $f$ we just note that if $n$ is large then in $2 \exp \left(\lambda^{n}\right) \leqq|z| \leqq \frac{1}{2}$ $\exp \left(\lambda^{n+1}\right)$, we have $H(z)=h(z)$. So for $3 \exp \left(\lambda^{n}\right) \leqq|z| \leqq \frac{1}{3} \exp \left(\lambda^{n+1}\right)$, we have

$$
\log |f(z)| \leqq \log M(|z|(1+o(1)), h)=0(\log |z|)^{1+\delta}
$$

which proves (5.2), and completes the proof of Lemma 3.

\section{Proof of Theorem 1}

Given $\sigma$ with $1 / 2<\sigma \leqq 1$, we first choose $\rho$ and $\delta$ with $1 / 2<\rho<1$ and $0<\delta<1$ and $\rho(1+\delta)=\sigma$. We choose $\lambda>1$ so that $\lambda^{\delta}$ is an integer, and such that (5.1) is satisfied. Now let the sequences $\left(\theta_{k}\right),\left(\alpha_{k}\right),\left(n_{k}\right)$ and $\left(\varepsilon_{k}\right)$ be as in the statement of Lemma 2. Since (3.1) is satisfied, we can, by Lemma 1 , find pairwise disjoint infinite sets $T_{k}$ of non-negative integers such that each $T_{k}$ is an arithmetic progression modulo $p_{k}$, where $p_{k} \leqq 2 / \alpha_{k}$. We now apply Lemma 3 , to construct an entire function $f$ such that (5.2) holds, and such that for some sequence $\left(B_{n}\right)$ satisfying (5.3) the following holds. If $n \geqq n^{*}$, if $n$ is in $T_{k}$ and $\lambda^{n} \geqq \log ^{+}\left|a_{k}\right|$, then (5.4) holds for all $z$ with $\left|z-B_{n}\right|<1$.

Now we define the constants $\beta_{k, n}$ as follows. Given $k \geqq 0$ and $n \geqq n^{*}$, let $m$ be the largest member of $T_{k}$ such that

$$
\lambda^{m}+1 \leqq \varepsilon_{k} \lambda^{n \rho}
$$

and set $\beta_{k, n}=B_{m}$. If no such $m$ exists, or if $k \geqq 0$ and $0 \leqq n<n^{*}$, we set $\beta_{k, n}=0$. Using Lemma 2 we construct an entire function $g$ of at most order $\rho$, mean type, such that if $n \geqq \max \left\{n_{k}, N_{0}\right\}$ we have 


$$
\left|g(z)-\beta_{k, n}\right|<1
$$

for

$$
\lambda^{n} \leqq|z| \leqq \lambda^{n+1},\left|\arg z-(-1)^{n} \theta_{k}\right| \leqq 2 \alpha_{k} .
$$

We set $F(z)=f(g(z))$. It follows at once, using (5.2), that

$$
T(r, F)=0\left(r^{\sigma}\right) .
$$

Now suppose that $k \geqq 0$, and that $n$ is large compared to $k$. Then for $z$ satisfying (6.3), we have $\left|g(z)-B_{m}\right|<1$, where $m$ is as defined by (6.1). Now (5.4) gives, for such $z$, provided $n$ is large enough,

$$
\log \left|F(z)-a_{k}\right|<-c \lambda^{m(1+\delta)} .
$$

But $T_{k}$ is an arithmetic progression modulo $p_{k}$, so that if $n$ is large enough,

$$
\lambda^{m} \geqq\left(\varepsilon_{k} \lambda^{n \rho}-1\right) \lambda^{-2 / \alpha_{k}} \geqq \frac{1}{2} \varepsilon_{k} \lambda^{n \rho} \lambda^{-2 / \alpha_{k}} .
$$

Denoting by $d_{1}, d_{2}, \ldots$ positive constants which do not depend on $n$ or $k,(6.5)$ now gives, for $z$ satisfying (6.3),

$$
-\log \left|F(z)-a_{k}\right|>d_{1} \lambda^{n \rho(1+\delta)}\left(\varepsilon_{k}\right)^{d_{2}} \lambda^{-d_{3} / \alpha_{k}}>d_{4}|z|^{\sigma}\left(\varepsilon_{k}\right)^{d_{2}} \lambda^{-d_{3} / \alpha_{k}} .
$$

Thus $F$ has order $\sigma$, mean type, each $a_{k}$ is a deficient value for $F$, and, using (3.2),

$$
\left(\log 1 / \delta\left(a_{k}, F\right)\right)^{-1} \geqq d_{5} \alpha_{k} .
$$

Concluding Remarks. The estimate (6.6) shows that the deficiencies tend to zero at a rate comparable to that in Arakelyan's construction [1], this rate being essentially only determined by (3.1).

It does not seem possible to prove by the present method that the $a_{k}$ are the only finite deficient values. In Eremenko's extension of Theorem B (which also uses the Teichmüller-Belinskii theorem) it is shown that the function $G$ may be constructed with the following property. There exist arbitrarily large circles on which, with the exception of a set of arbitrarily small angular measure, $G$ is either large or is close to one of the $a_{k}$. For our problem, if $A$ is not one of the $a_{k}$, we would need to estimate the proximity of $F$ to $A$ in terms of the proximity of $g$ to the roots of $f(w)=A$.

Acknowledgements The author would like to acknowledge valuable conversations with his colleagues Günter Frank and D. A. Burgess. 


\section{REFERENCES}

1. N. U. Arakelyan, Entire functions of finite order with an infinite set of deficient values, (Russian) Dokl. Akad. Nauk SSSR 170 (1966) No. S, (Translation) Soviet Math. Dokl. 7 (1966), 1303-1306.

2. D. Drasin and W. K. Hayman, Value distribution of functions meromorphic in an angle, Proc. London Math. Soc. (3) 48 (1984), 319-340.

3. A. Edrel, and W. H. J. Fuchs, Valeurs déficientes et valeurs asymptotiques des fonctions méromorphes, Comment. Math. Helv. 33 (1959), 258-295.

4. A. E. ERemenko, On the set of defect values of a finite order entire function, (Russian) Ukrain. Mat. Zh. 39 (1987), 295-299, (Translation) Ukrainian Math. J. 39 (1987), 225-228.

5. R. Goldstein, On factorization of certain entire functions, J. London Math. Soc. (2) 2 (1970), 221-224.

6. R. Goldstein, On factorization of certain entire functions II, Proc. London Math. Soc. (3) 22 (1971), 483-506.

7. F. Gross, Factorization of Meromorphic Functions (Mathematics Research Center, Naval Research Laboratory, Washington D.C., 1972).

8. W. K. HaYman, Slowly growing integral and subharmonic functions, Comment Math. Helv. 34 (1960), 75-84.

9. W. K. Hayman, Meromorphic Functions (Oxford at the Clarendon Press, 1964).

10. W. K. Hayman and J. Miles, On the growth of a meromorphic function and its derivatives, Complex Variables 12 (1989), 245-260.

11. O. Lehto and K. I. ViRTANen, Quasiconformal Mappings in the Plane (2nd Edition, Springer Berlin, 1973).

12. O. Martio, S. Rickman and J. VäısÄLÄ, Distortion and singularities of quasiregular mappings, Ann. Acad. Sci. Fenn. AI 465 (1970), 1-13.

13. S. N. Mergelyan, Uniform approximation to functions of a complex variable, Uspekhi Mat. Nauk 7, 2 (48) (1952), 31-122, (Translation) Amer. Math. Soc. Transl. Ser. I, No. 3, Providence RI 1954.

14. S. N. Mergelyan, On the completeness of systems of analytic functions, (Russian) Uspekhi Mat. Nauk 8 (1953), no. 4 (56), 3, 3-63 (Translation) Amer. Math. Soc. Transl. Ser. 2, 19 (1962), 109.

15. S. Topplla, On Nevanlinna's characteristic functions of entire functions and their derivatives, Ann. Acad. Sci. Fenn. AI 3 (1977), 131-134.

16. S. ToppiLA, On the characteristic of meromorphic functions and their derivatives, J. London Math. Soc. (2) 25 (1982), 261-272.

17. C. C. YANG, Some aspects of factorization theory-a survey, Complex Variables 13 (1989), $133-142$.

Department of Mathematics

UNIVERSITY OF NOTTINGHAM

NotTINGHAM NG7 2RD 